\title{
Brief mindfulness training alters causal brain connections in $\mathrm{mTBI}$
}

\author{
Rongxiang Tang ${ }^{1}$, Yi-Yuan Tang ${ }^{2^{*}}$ \\ From 24th Annual Computational Neuroscience Meeting: CNS*2015 \\ Prague, Czech Republic. 18-23 July 2015
}

Traumatic brain injury (TBI) is a significant cause of disability in the United States and the mild TBI (mTBI) is the most prevalent. Previous research indicates the positive effect of mindfulness training on symptoms of chronic mTBI such as cognitive functioning and emotion [1]. However it remains unclear which brain regions play a crucial role in $\mathrm{mTBI}$ recovery and the causal relationship among these regions. Here we apply a dynamic causal modeling (DCM) in resting state fMRI [2] to demonstrate the causal relationships among the core regions involved in $\mathrm{mTBI}$.

Fourteen veteran students with mTBI were recruited through campus advertisements. We used 2 weeks of integrative body-mind training as mindfulness intervention, previously reported in our series of randomized studies $[3,4]$. All data were collected using a 3-Telsa Siemens Skyra scanner. Functional data were processed using the Data Processing Assistant for Resting-State fMRI, which is based on SPM and Resting-State fMRI Data Analysis Toolkit. Based on literature, we specified four regions of interest within default mode network (DMN) - medial prefrontal cortex (mPFC), posterior cingulate cortex (PCC), and bilateral inferior parietal lobule (Left IPL and Right IPL), same regions and coordinates as in previous SDCM studies [2,5]. Based on SPM12, we estimated and specified the DCM for each subject and later compared the differences of effective connectivity before and after mindfulness. Our results suggest the different causal relationships following mindfulness training. Specifically, after training there was a significant decrease in the strength of excitatory input from $\mathrm{mPFC}$ to $\mathrm{PCC}$, and a significant increase in

\footnotetext{
* Correspondence: yiyuan.tang@ttu.edu

2Department of Psychological Sciences, Texas Tech University, Lubbock, TX 79409, USA

Full list of author information is available at the end of the article
}

the strength of inhibitory input from mPFC to LIPL (all $\mathrm{p}<0.05)$.

\section{Conclusions}

Resting DCM can differentiate the causal brain connections before and after mindfulness training, and provide insight into the brain mechanisms of altered DMN dynamics underlying $\mathrm{mTBI}$ recovery, suggesting the changes in information flow in these distributed systems involved in mTBI intervention.

\section{Acknowledgements}

This work was supported by the Office of Naval Research.

\section{Authors' details}

${ }^{1}$ Department of Psychology, The University of Texas at Austin, Austin, TX 78712, USA. ²Department of Psychological Sciences, Texas Tech University, Lubbock, TX 79409, USA.

Published: 18 December 2015

\section{References}

1. Azulay J, Smart CM, Mott T, Cicerone KD: A pilot study examining the effect of mindfulness-based stress reduction on symptoms of chronic mild traumatic brain injury/postconcussive syndrome. J Head Trauma Rehabil 2013, 28:323-31.

2. Razi A, Kahan J, Rees G, Friston KJ: Construct validation of a DCM for resting state fMRI. Neuroimage 2015, 106:1-14.

3. Tang $Y Y$, et al: Short-term meditation training improves attention and self-regulation. Proceedings of the National Academy of Sciences, USA 2007, 104:17152-17156.

4. Tang YY, et al: Central and autonomic nervous system interaction is altered by short term meditation. Proceedings of the National Academy of Sciences, USA 2009, 106:8865-70.

5. Di X, Biswal BB: Identifying the default mode network structure using dynamic causal modeling on resting-state functional magnetic resonance imaging. Neuroimage 2014, 86:53-9.

doi:10.1186/1471-2202-16-S1-P247

Cite this article as: Tang and Tang: Brief mindfulness training alters causal brain connections in mTBI. BMC Neuroscience 2015 16(Suppl 1): P247. 